Research Article

\title{
Evaluating the Behavior of Embankments after Widening considering Soil Consolidation and Splicing Mode
}

\author{
Xiaobo Xing, ${ }^{1}$ Zhenhao Bao $\mathbb{D}^{2},{ }^{2}$ and Wei $\mathrm{Li}^{1}$ \\ ${ }^{1}$ Shandong Hi-speed Tsingtao Construction Management Group Co., Ltd., Tsingtao 266300, Shandong, China \\ ${ }^{2}$ School of Civil Engineering, Shandong University, Jinan 250061, Shandong, China \\ Correspondence should be addressed to Zhenhao Bao; bzh1998@mail.sdu.edu.cn
}

Received 15 February 2021; Revised 7 October 2021; Accepted 1 December 2021; Published 16 December 2021

Academic Editor: Wenjun Zhu

Copyright () 2021 Xiaobo Xing et al. This is an open access article distributed under the Creative Commons Attribution License, which permits unrestricted use, distribution, and reproduction in any medium, provided the original work is properly cited.

Increased traffic volume has made it necessary to increase highway capacities by widening embankments and pavements. Differential settlement of foundation consolidation and rational utilization of existing embankments are the main problems encountered in road reconstruction. In this paper, the finite difference method is used to simulate the construction process of using the existing embankments directly in the reconstruction project of expressway, and the fluid-solid coupling model of foundation settlement is established to calculate the differential settlement between foundation and subgrade. The influence of road widening mode and embankment height on differential settlement is analyzed. The mechanical response of subgrade under differential settlement is simulated and the law of uneven settlement of main reconstruction forms is investigated. The dynamic response of existing embankments soil under the action of rammer is systematically evaluated. The results show that, with the increase of consolidation time, the differential settlement is gradually obvious at the junction of the new and existing embankments, and there is a possibility of landslide along the junction of new embankments, which should be dealt with in engineering. The smaller the height difference between the existing foundation and the new foundation is, the more the position of the maximum settlement point of the foundation moves towards the new foundation. The greater the height of the new subgrade is, the greater the uneven settlement is. The criterion based on the single rammed energy and compaction stopping standard is proposed to determine the reinforcement depth of existing embankment.

\section{Introduction}

Widening of embankments has been increasingly adopted in practice to increase highway capacities due to demand for higher traffic volume than previously designed. The 1989 Government's White Paper "Roads for Prosperity" [1] indicated that "about $60 \%$ of the motorway network in England as well as some truck roads will need to be widened by the provision of additional lanes." Adding a new embankment adjacent to the existing embankment induces not only additional stresses and deformations under the widened portion but also those under the existing embankment. Differential settlement may develop between and within the new and existing embankments. This often causes pavement distress, such as longitudinal cracks or drop-off of pavement sections $[2,3]$. Therefore, it is necessary to do research on utilization of existing embankments and differential settlement control in widening of expressway [4-6].

Up to now, a lot of research programs have been fruitfully developed on this subject. By employing a large centrifugal tester and finite element program, Allersrma et al. [7] and Vos et al. [8] analyzed the stress distribution and deformation law of roadbed widening during construction by means of model test, numerical simulation, and field test. Zhou and Zhang [9] and Qian and Ling [10] used finite element calculation method to analyze the law of differential settlement of existing and new embankments in reconstruction and expansion project, studied the influence of different widening ways and widths on differential settlement, and explored the significant influence of differential settlement on the structural performance and service performance of existing roadbed and road surface. Similarly, Li 
et al. [11] used PLAXIS and a small centrifugal tester to analyze the destabilization of widened subgrade and to evaluate two different methods of embankment filling. Huang et al. [12] conducted their laboratory tests on a widened subgrade. In the tests, a layer of soluble chemical fertilizer at the bottom of embankment fill was employed. It was to be resolved by water. By doing so, the differential settlement was simulated. Weng and Wang [13] designed large-scale model test to investigate the influence of differential settlement on pavement structure of widened roads.

Even though widening of embankments has been increasingly adopted in practice, so far very limited guidance for design has been available for widening projects. Chen et al. [14] and Zhang et al. [15-17] used numerical simulation method to monitor the changes of stress in roadbed and proposed corresponding control methods. Yang [18] and Nie et al. [19] discussed design thinking and construction control of soft soil foundation in reconstruction and extension construction of the Shen-Da Expressway. A boundary condition is imposed to the finite element model of pavement structure in order to simulate differential settlement on the pavement structure. Cheng et al. [20] and Zhao et al. [21] proposed a differential settlement control standard considering flexural strength of pavement materials and fatigue damage performance of pavement structure.

From the above analysis, it should be pointed out that the problem of uneven settlement control of existing and new embankments has not been well solved. It is mainly based on the two following reasons: (a) after years of consolidation, the compactness of the existing natural foundation is much higher than that of the new natural foundation, and the stiffness and strength are mainly increased mechanically. (b) The load of the newly filled embankment becomes eccentric load, as shown in Figure 1. In the embankment reconstruction, this part is the main load that causes the settlement of the foundation.

In this paper, based on the Wei-Ru expressway reconstruction project, the differential settlement caused by consolidation is calculated by using the finite difference software FLAC, and the law of differential settlement is analyzed. Not only the uneven settlement caused by the general road widening but also the settlement caused by the roadbed elevation is considered. The fluid-solid coupling model of foundation settlement is established to calculate the differential settlement between foundation and subgrade. Based on the study of dynamic compaction parameters of existing embankments, the determination of single click compaction energy and the standard of stop compaction are proposed as the standards for determining the reinforcement depth of existing embankments. At present, people's research on this is relatively simple, so this paper will explore it.

\section{Project Overview}

Subgrade settlement includes two aspects: one is the final settlement, and the other is the settlement with time change law, that is, the consolidation of soil. For the final settlement,

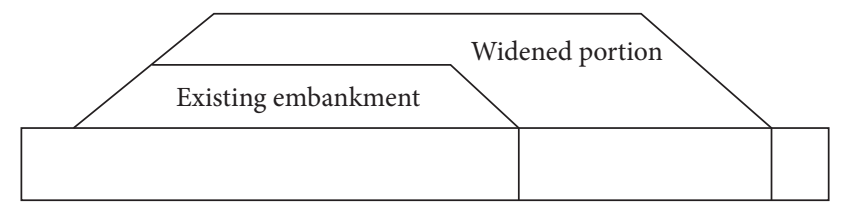

Figure 1: Schematic diagram of the new and existing embankments.

the current theory is more perfect, but the law of settlement with time is still the key and difficult problem in the engineering field. The traditional calculation method of the final settlement mainly divides the settlement into three parts: instantaneous settlement, consolidated settlement, and secondary consolidated settlement; namely, $S=S_{\mathrm{d}}+S_{\mathrm{c}}+S_{\mathrm{S}}$. The secondary consolidation settlement is generally small, so it is not considered.

The renovation project upgraded the original grade one road to a highway. The foundation surface of the test section was more than $2 \sim 3 \mathrm{~m}$ of clay soil containing sand and gravel, and the paleochannel sand deposits existed generally below $2 \sim 3 \mathrm{~m}$. As a banded structure, the deformation of embankment mainly occurs on the cross section, which can be regarded as a plane strain problem. In order to analyze the influence of widening mode and embankment height on differential settlement of foundation, three working conditions are mainly calculated (see Figure 2). In working conditions 1 and 2, the width of existing embankment is $18 \mathrm{~m}$ and the height is $3 \mathrm{~m}$. The new embankment is $28 \mathrm{~m}$ wide and $6 \mathrm{~m}$ high. The slope is $1: 1.5$. Working condition 1 is unilateral widening; working condition 2 is bilateral broadening; working condition 3 also widens both sides, but the height of the existing embankment is $6 \mathrm{~m}$.

The mechanical parameters of the existing embankment and the new and existing foundation soil were obtained through field core samples and laboratory tests (see Table 1), from which it can be seen that the compaction degree of the existing embankment is far lower than the current standard of compaction degree of the expressway embankment.

\section{Establishment of Fluid-Solid Coupling Model}

3.1. Governing Equations of Fluid-Solid Coupling in Porous Media. The following equations must be satisfied when fluid-solid coupling calculation of porous media is carried out.

3.1.1. Equilibrium Equation. For small deformation, the equilibrium equation of the fluid particle is

$$
-q_{i, j}+q_{v}=\frac{\partial \xi}{\partial_{t}}
$$

where $q_{i, j}$ is the seepage velocity $(\mathrm{m} / \mathrm{s}) ; q_{v}$ is fluid source strength $(1 / \mathrm{s}) ; \xi$ is the change in fluid volume per unit volume of porous media.

$$
\frac{\partial \xi}{\partial_{t}}=\frac{1}{M} \frac{\partial_{P}}{\partial_{t}}+\alpha \frac{\partial_{z}}{\partial_{t}}-\beta \frac{\partial_{T}}{\partial_{t}}
$$




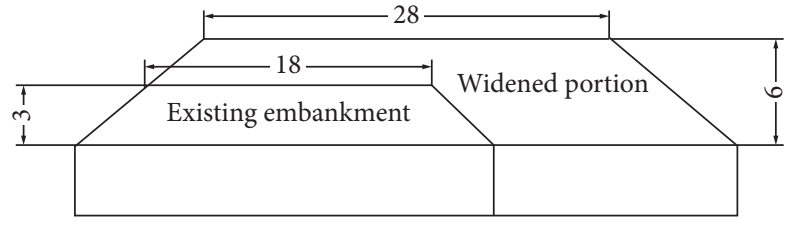

(a)

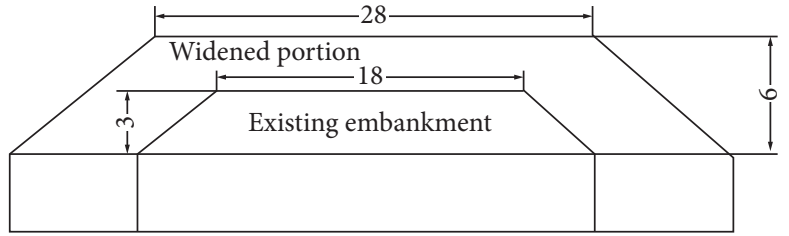

(b)

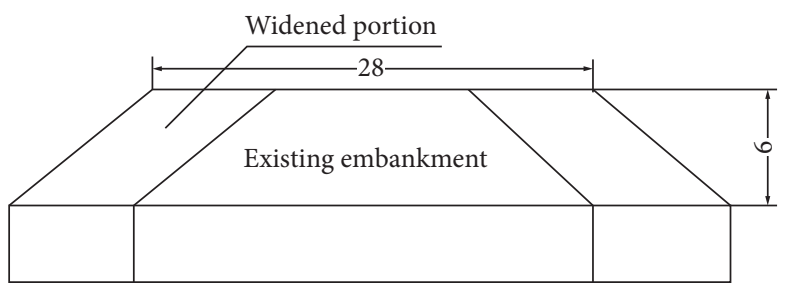

(c)

Figure 2: Schematic diagram of three calculation conditions (unit: $\mathrm{m}$ ).

TABLE 1: Soil mechanical parameters corresponding to the existing embankments height $H=4 \mathrm{~m}$.

\begin{tabular}{|c|c|c|c|c|c|c|}
\hline Location & Soil depth $(\mathrm{m})$ & Compactness (\%) & $c(\mathrm{kPa})$ & $\varphi\left(^{\circ}\right)$ & $k$ & $n$ \\
\hline \multirow{3}{*}{ The existing embankment } & 0.9 & 80.0 & 3.0 & 35.7 & 331.1 & 0.51 \\
\hline & 2.3 & 68.6 & 10.0 & 31.6 & 251.2 & 0.62 \\
\hline & 4.0 & 69.0 & 10.0 & 29.3 & 218.8 & 0.66 \\
\hline \multirow{3}{*}{ The existing foundation } & 5.7 & & 29.0 & 23.2 & 128.8 & 0.58 \\
\hline & 7.1 & - & 19.0 & 27.9 & 173.8 & 0.64 \\
\hline & 8.2 & & 9.0 & 33.3 & 316.2 & 0.53 \\
\hline \multirow{3}{*}{ The new foundation } & 0.2 & & 14.0 & 32.0 & 208.9 & 0.61 \\
\hline & 1.4 & - & 23.0 & 23.3 & 138.0 & 0.64 \\
\hline & 3.0 & & 24.0 & 26.2 & 173.8 & 0.66 \\
\hline \multirow{3}{*}{ The new embankment } & & 93.0 & 28.0 & 30.1 & 320.4 & 0.70 \\
\hline & Earth field & 94.0 & 26.2 & 33.2 & 341.8 & 0.72 \\
\hline & & 96.0 & 24.6 & 35.0 & 355.2 & 0.78 \\
\hline
\end{tabular}

where $M$ is modulus of $\operatorname{Biot}\left(\mathrm{N} / \mathrm{m}^{2}\right) ; P$ is pore pressure; $\alpha$ is coefficient of Biot; $\xi$ is volume strain; T is temperature; $\beta$ is the coefficient of thermal expansion considering fluid and particle.

In this paper, without considering the thermal expansion factor, equation (2) can be simplified as

$$
\frac{\partial \xi}{\partial_{t}}=\frac{1}{M} \frac{\partial_{P}}{\partial_{t}}+\alpha \frac{\partial_{z}}{\partial_{t}}
$$

3.1.2. Equation of Motion. The motion of fluid is described by Darcy's law. For the case where the density of homogeneous and isotropic solid and fluid is constant, the equation is as follows:

$$
q_{i}=-k\left[p-p_{f} x_{j} g_{j}\right]_{, i}
$$

where $k$ is the permeability coefficient of the medium $\left[\mathrm{m}^{4} /\right.$ $(\mathrm{N} \cdot \mathrm{s})] ; p_{f}$ is fluid density $\left(\mathrm{kg} / \mathrm{m}^{3}\right) ; g_{i}(i=1,2,3)$ are the three components of the acceleration of gravity $\left(\mathrm{m} / \mathrm{s}^{2}\right)$.
3.1.3. Constitutive Equation. The change of volumetric strain causes the change of pore pressure of fluid, and, conversely, the change of pore pressure also causes the occurrence of volumetric strain. The incremental form of the constitutive equation of porous media is

$$
\Delta \delta_{i j}+\alpha \Delta p \delta_{i j}=H_{i j}^{*}\left(\delta_{i j} \Delta \varepsilon_{i j}-\Delta \varepsilon_{i j}^{T}\right)
$$

In the above equation, $\Delta \delta_{i j}$ is stress increment; $H_{i j}^{*}$ is a given function; $\Delta \varepsilon_{i j}$ is the total strain; $\Delta \varepsilon_{i j}^{T}$ is the temperature strain.

3.1.4. Compatibility Equation. The relationship between strain rate and velocity gradient is

$$
\varepsilon=\frac{\left(v_{L, J}+v_{J, L}\right)}{2},
$$

where $v_{L}$ is the velocity of a certain point in the medium. 
3.2. Establishment of Constitutive Model. The constitutive model used in the calculation is Mohr-Coulomb model, in which the elastic modulus is calculated as follows:

$$
E=k p_{a}\left(\frac{\sigma_{3}}{P_{a}}\right)^{n} \text {. }
$$

In the formula, $k$ and $n$ are two mechanical parameters of Duncan-Chang model (see Table 1). $P_{a}$ is atmospheric pressure; $\sigma_{3}$ is the confining pressure; Poisson's ratio $\mu=0.25$. In calculation, the vertical stress at the top of the element is taken as the sum of the vertical stress generated by the dead weight of the overlying soil and the pavement (surface layer and base layer).

The isotropic fluid model and Darcy's law model were selected in the calculation of this paper. The fluid parameters include porosity ratio and permeability coefficient. The porosity ratio is 0.456 and the permeability coefficient is $7.75 \times 10^{-10} \mathrm{~m} / \mathrm{s}$ when the foundation depth is $0 \sim 1.5 \mathrm{~m}$. The pore ratio below $1.5 \mathrm{~m}$ is 0.386 and permeability coefficient is $7.34 \times 10^{-12} \mathrm{~m} / \mathrm{s}$. According to the site situation, the groundwater level is set at $0.8 \mathrm{~m}$ below the foundation.

The Mohr-Coulomb elastic-plastic model commonly used in geotechnical engineering is used as the material model of embankment and foundation soil in the calculation. The model can be expressed as follows:

$$
F=\sigma_{1} \tan ^{2}\left(\frac{\pi}{4}+\frac{\phi}{2}\right)-\sigma_{3}+2 c \tan \left(\frac{\pi}{4}+\frac{\phi}{2}\right)=0 .
$$

Before the construction of the road, the natural foundation has been consolidated for several years, and the settlement has been basically completed. The excess pore water pressure in the foundation is very small, and it will not sink again under the action of its own weight. In order to simulate this state, before simulating the settlement of foundation caused by the deadweight of roadbed, the foundation is first consolidated under its deadweight. The displacement of the foundation is initialized to zero before the embankment is filled.

\section{Study on Differential Settlement of Foundation under Different Working Conditions}

4.1. Results and Analysis of Differential Settlement of Foundation. Figure 3 shows the settlement and water pressure results at the midpoint of foundation under 3 working conditions. It can be seen from Figure 3 that the construction of new embankment and surface structure causes instantaneous excess pore water pressure and instant consolidation in the foundation. With the increase of time, the excess pore water pressure dissipates gradually, and the vertical displacement of the foundation increases gradually, which is the process of consolidation. Finally, the pore water pressure tends to be stable and the consolidation settlement gradually becomes stable.

Figures 4-6 show the ground surface settlement of the new embankment at 6 months and 1 year after construction
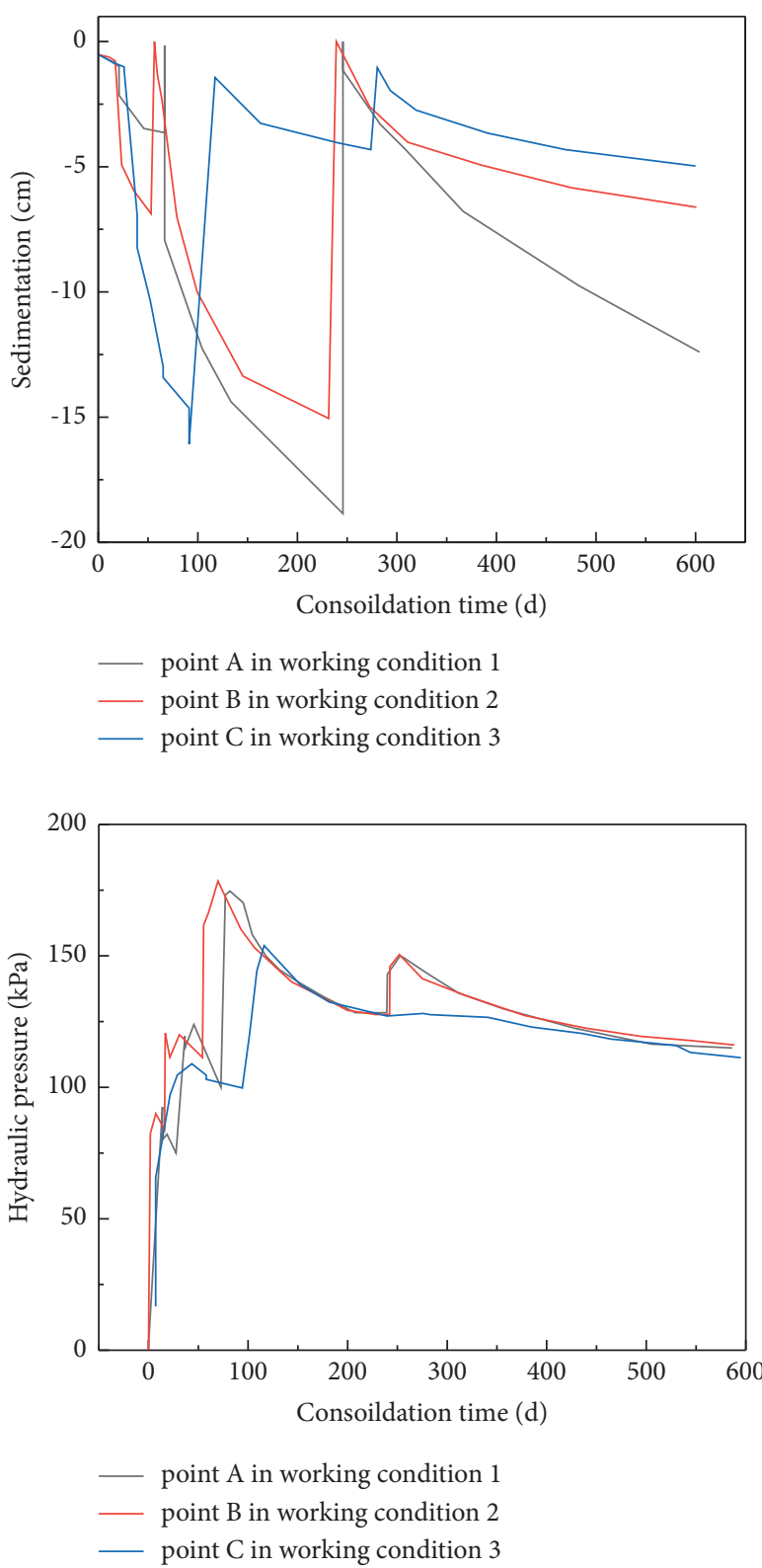

Figure 3: Settlement curve and water pressure curve at the middle point of foundation under different working conditions.

under three working conditions. The following can be seen from Figures 4-6:

(1) Compared with unilateral widening, the peak value of foundation settlement decreases slightly. This is mainly because the stress on both sides of the existing foundation is balanced due to bilateral widening. The deadweight load of the new subgrade increases the additional stress in the middle of existing embankments, which leads to greater settlement in the centre of existing embankments. However, the widening of one side causes the foundation to be affected by the eccentric force, which is mainly the consolidation deformation of the new foundation. 


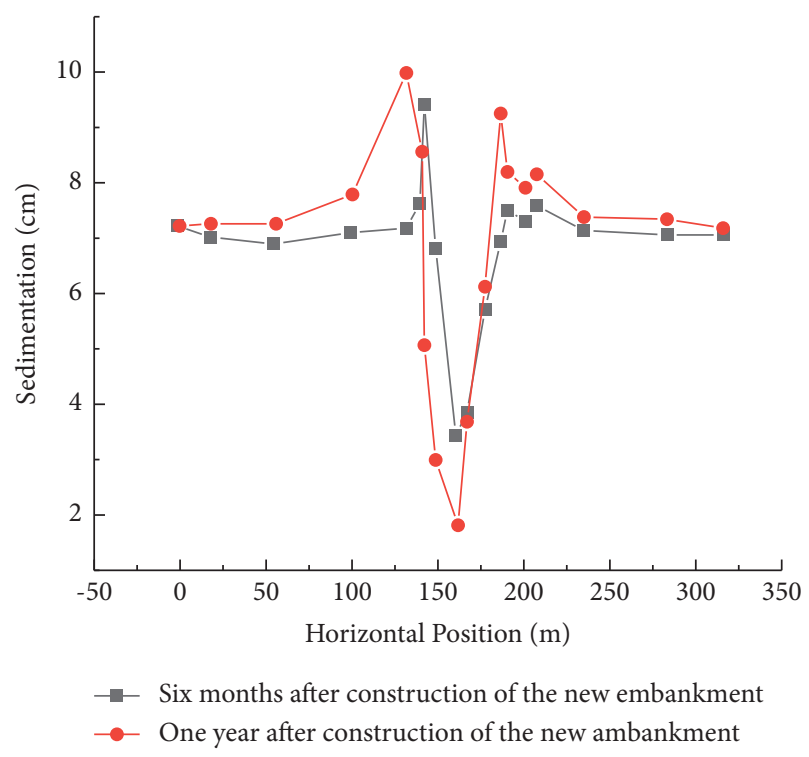

FIgURE 4: Sedimentation of foundation surface (condition 1).

(2) Both sides are widened. Compared with Figures 5 and6, the settlement generated by working condition 3 is relatively small. That is to say, with the height of the new embankment unchanged, the maximum settlement of the foundation decreases gradually with the increase of the height of the existing embankment. This is because the higher the existing embankments, the greater the backpressure effect on the new natural foundation.

(3) The differential settlement of the new foundation mainly occurs at the slope of the existing embankment, which is the junction of the existing and new embankment. So, the project should focus on the new and existing embankment.

\subsection{Research on Foundation Settlement under Main Working} Conditions. The settlement was calculated by using FLAC ${ }^{3 \mathrm{D}}$ finite difference program, and the plastic failure and plastic flow were simulated by mixed discrete method. This method is more accurate and reasonable than the "discrete integration method" commonly used in the finite element method. Even if the simulated system is static, the dynamic motion equation is still used, which makes FLAC ${ }^{3 \mathrm{D}}$ simulation of physically unstable processes without numerical barriers. The "explicit solution" scheme is adopted, so the time taken to solve the nonlinear stress-strain relation is almost the same as that of the linear constitutive relation, while the implicit solution scheme will take a longer time to solve the nonlinear problem, and there is no need to store the stiffness matrix. This means that multielement structures can be solved with medium memory capacity, and the simulation of large deformation problems consumes almost no more computational time than that of small deformation problems, because no stiffness matrix has to be modified.

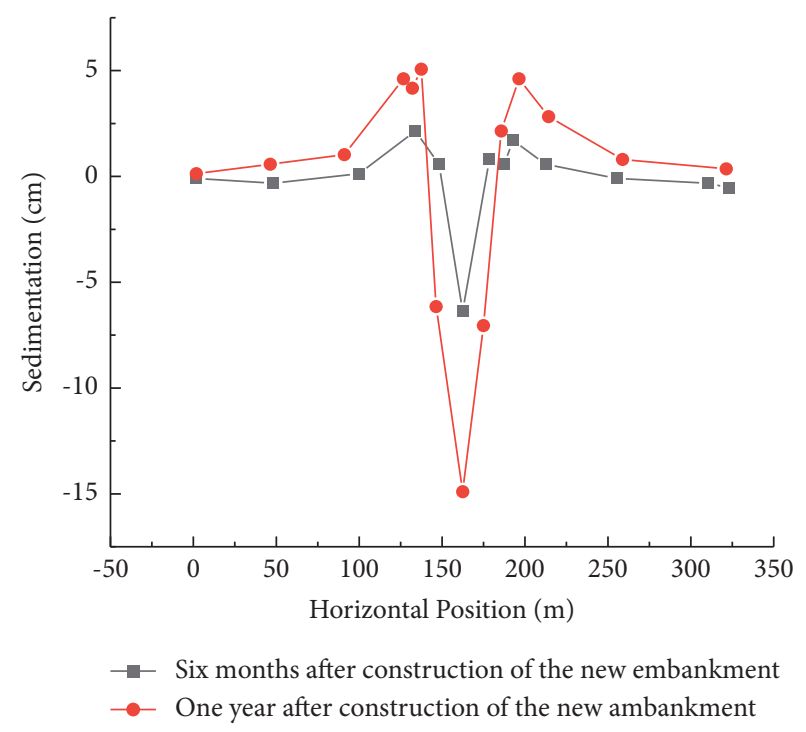

FIGURE 5: Sedimentation of foundation surface (condition 2).

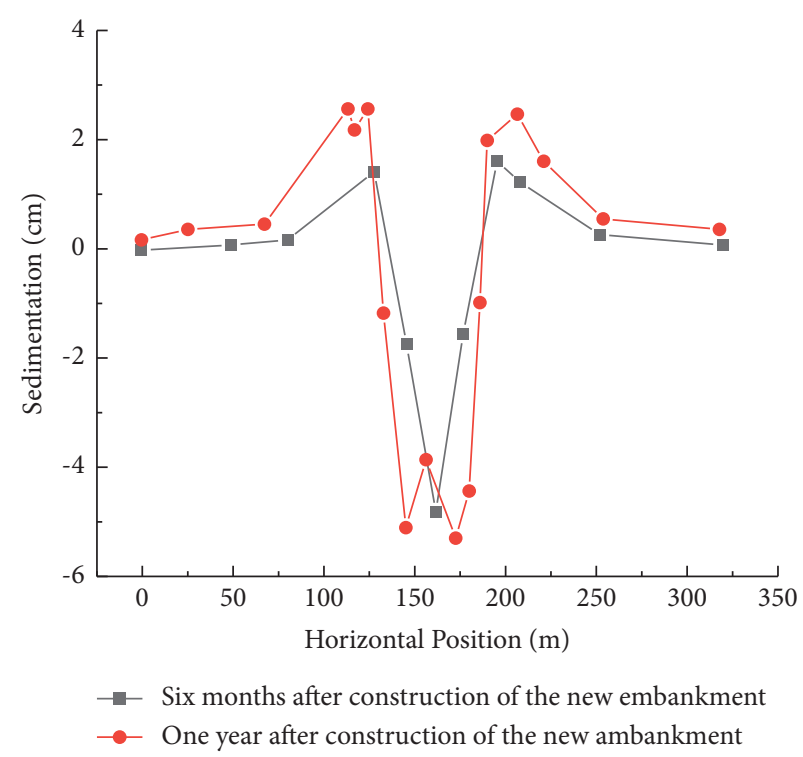

FIgURE 6: Sedimentation of foundation surface (condition 3).

As shown in Figure 1, the relative position of the existing and new roadbed is most likely to produce uneven settlement. This section carries out simulation calculation based on this widening form. Figure 7 shows the settlement diagram corresponding to the height of new embankment $h=6 \mathrm{~m}$ and the height of existing embankment $H=1 \mathrm{~m}$. Figure 8 shows the particle velocity vector diagram. It can be seen that the movement trend of soil particles to the new foundation is large, and there is a great difference in settlement at the junction of the new and existing subgrade. On the one hand, this is because the consolidation and settlement of the foundation under the weight of the existing embankment have been completed before. On the other 


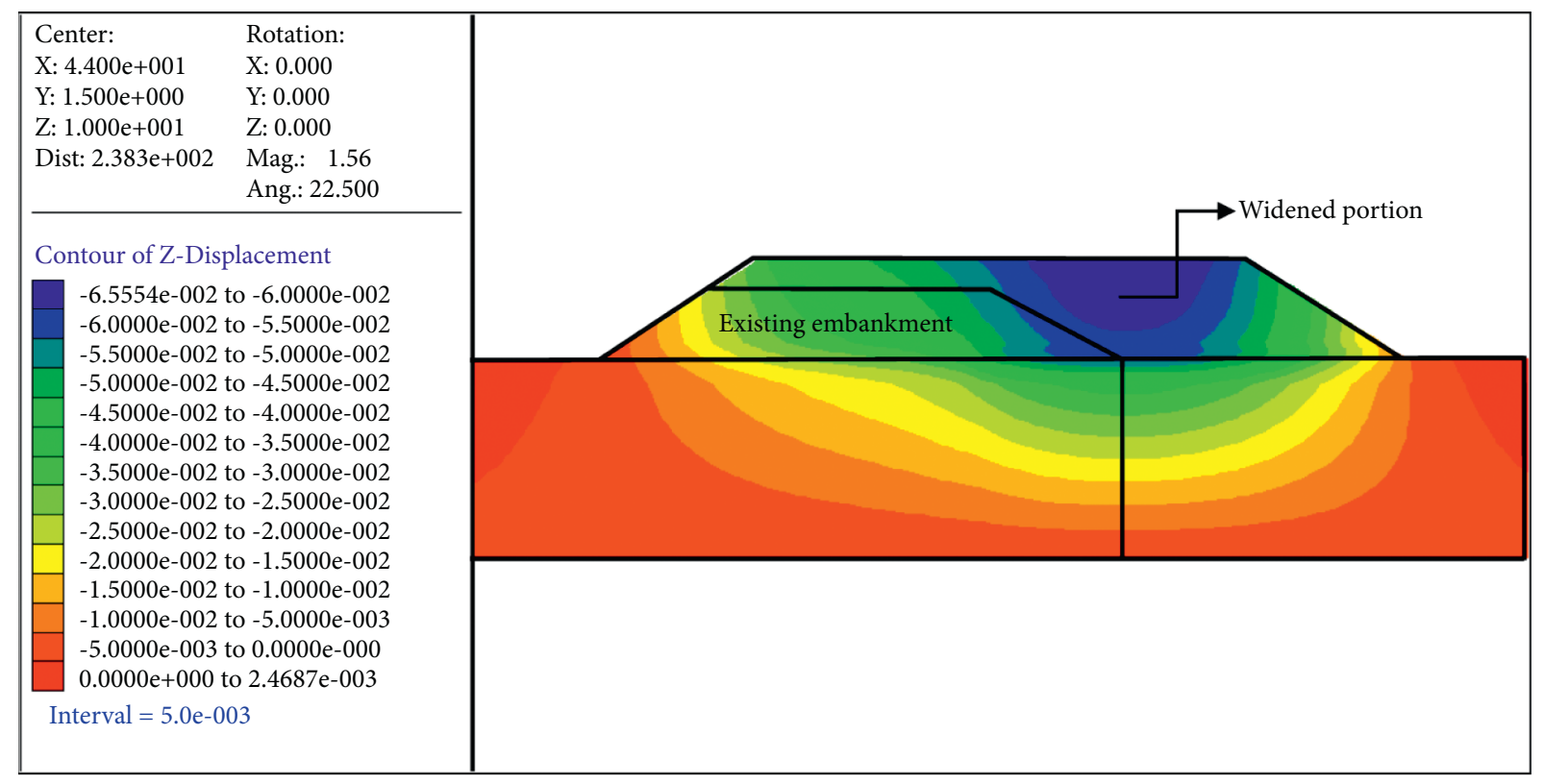

FIgURE 7: Settlement diagram.

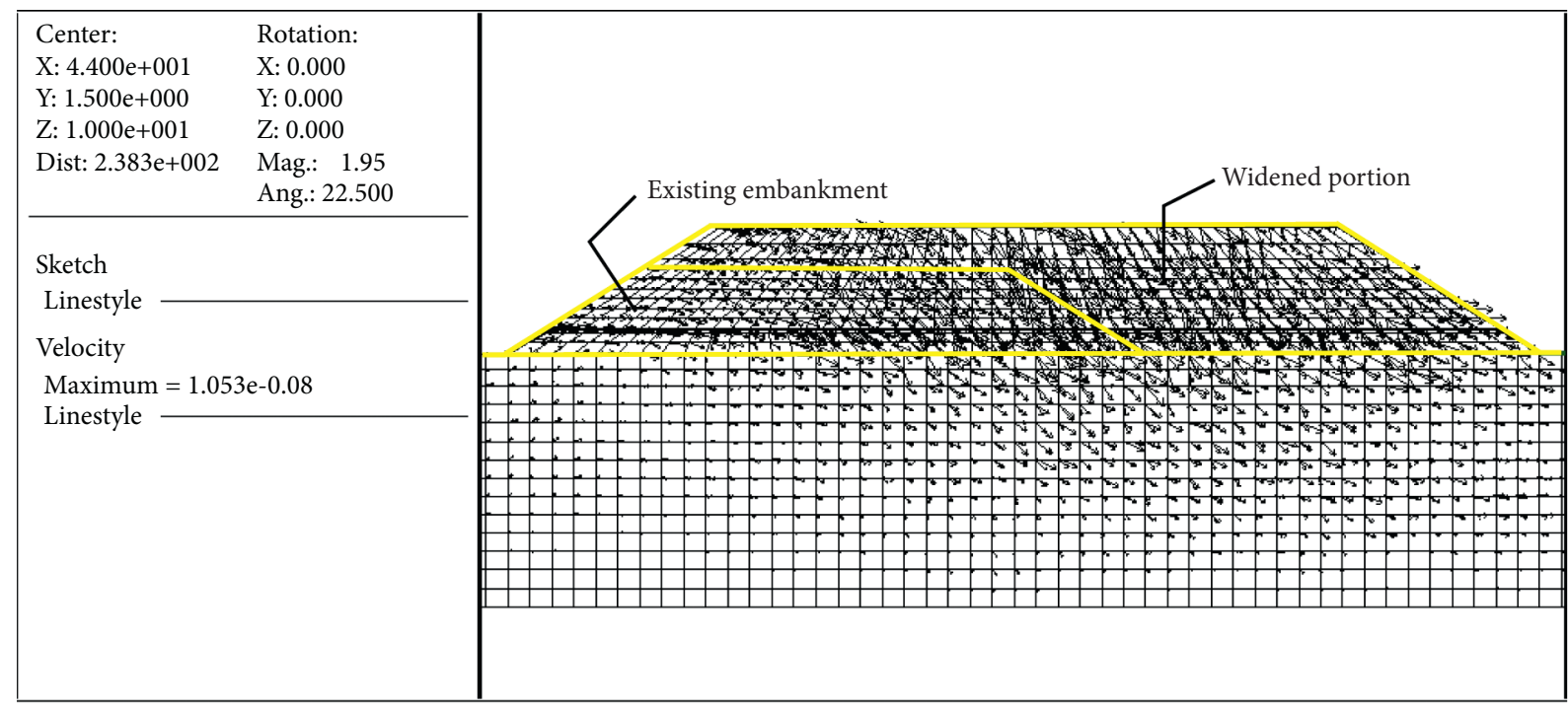

FIGURE 8: The particle velocity vector diagram.

hand, because the existing foundation has been consolidated for a long time, it has greater stiffness and strength than the new foundation.

Figure 9 shows the corresponding settlement curves of foundation and subgrade when $h=1 \mathrm{~m}$ and $H=6 \mathrm{~m}$. It can be seen that the settlement curve is asymmetric, and the settlement of subgrade is mainly caused by the settlement of foundation. The existence of the existing subgrade will make the settlement curve of the foundation surface have three characteristic points, $\mathrm{A}, \mathrm{B}$, and $\mathrm{C}$. In the absence of existing embankments, the curve will develop along $A B$. Because of the existence of existing embankments, the load that causes new settlement becomes eccentric load. Moreover, due to the different degree of consolidation of new and existing foundations, their stiffness is different, so that the $\mathrm{BC}$ curve becomes slow, while the AC curve becomes steep on the right slope of the existing embankment. When evaluating the settlement of road foundation, uneven settlement is often used as the evaluation index. The nonuniform settlement is generally expressed by the slope of the settlement curve, that is, the absolute settlement difference on the unit width. The embankment where the nonuniform settlement is large is easy to be destroyed. As can be seen from Figure 8, the maximum uneven settlement occurs on the right slope of the existing embankment, that is, at the junction of the existing and new embankment, so the junction is the key position of foundation treatment.

After the reconstruction, the width of the top surface of the new subgrade is $2 l$, and the transverse distance between the maximum settlement point of the foundation and the 


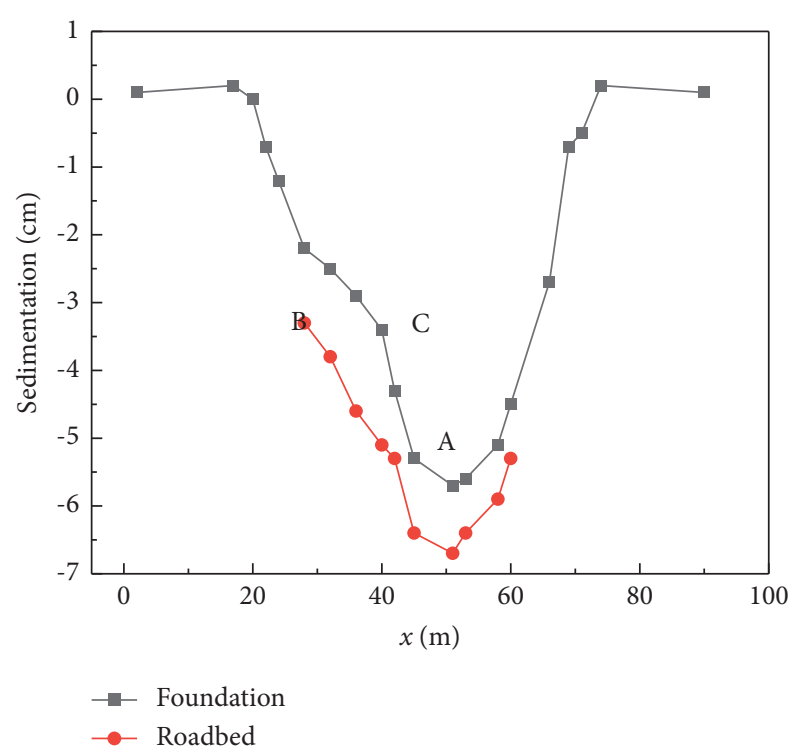

Figure 9: Settlement curve of foundation and subgrade.

median line of the subgrade is $x_{\mathrm{m}}$. The dimensionless $x_{\mathrm{m}} / l$ is introduced to represent the relative position of the maximum settlement point of the foundation. $X_{\mathrm{m}} / l=0$ indicates that the maximum settlement point is in the middle position of the new roadbed. $X_{\mathrm{m}} / l=1$ means that the maximum settlement point is at the right shoulder of the new roadbed. $X_{\mathrm{m}} / l=-1$ indicates that the maximum settlement point is at the left shoulder of the new roadbed. The only load that causes new deformation of foundation is the dead weight of the Shadowed part in Figure 1, which is a typical eccentric load. For the convenience of analysis, another dimensionless quantity-eccentricity $e(e=H / h)$ is introduced here to represent eccentricity. When $H=0$ and $e=0$, it means that the embankment is all newly filled embankment, with no eccentricity. When $H=h, e=1$, indicating that the eccentricity reaches the maximum, the existing embankment and the new embankment have the same height. Figure 10 shows the change curve of $x_{\mathrm{m}} / l$ with $e$ when $h=6 \mathrm{~m}$. It can be seen that when $e$ varies from 0 to $1.0,\left(x-x_{\mathrm{m}}\right) / l$ varies from 0 to 1.0 , which means that, with the increase of eccentricity, the position of the maximum settlement point of foundation gradually shifts to the right. Therefore, in order to reduce the uneven settlement of the foundation and subgrade, active measures should be taken to strengthen the new natural foundation and reduce the absolute settlement of the foundation.

From the perspective of foundation stiffness, the larger the difference between the compactness of the new foundation and the compactness of the existing foundation is, the greater the uneven settlement of the foundation will be; that is to say, the uneven settlement of the foundation is proportional to height $H$ of the existing subgrade. At the same time, the uneven settlement of foundation is related to the load eccentricity $e$, and the uneven settlement increases with the increase of $e$. In addition, the uneven settlement is also related to the average overburden load on the new or existing foundation, and the average overburden load can be

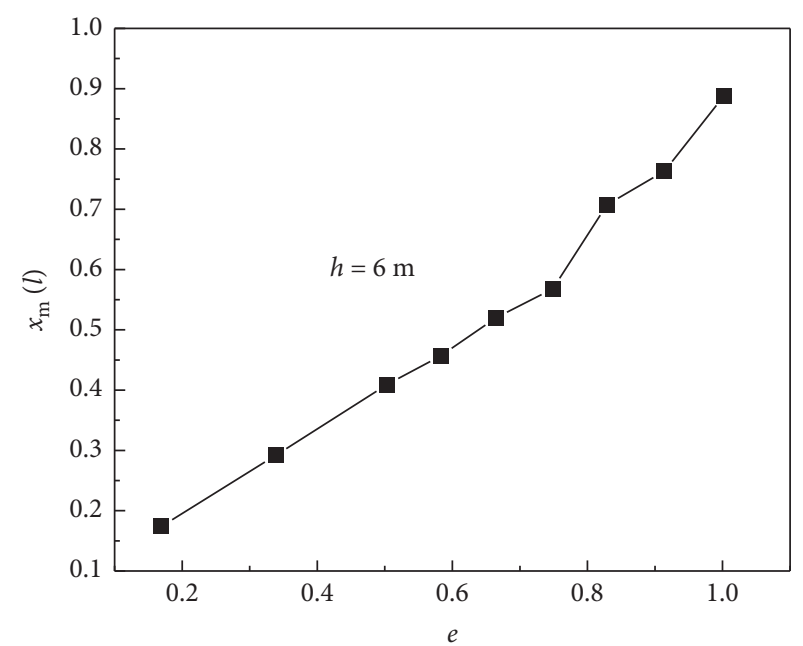

FIGURE 10: The relative position of the maximum settlement point of foundation varies with the degree of eccentricity.

expressed as $a h+b(h-H)$ ( $a$ and $b$ are positive coefficients). The larger the overburden load is, the greater the settlement is, and the greater the uneven settlement is. Of the three variables $H, e, a h+b(h-H)$, the first two are directly proportional to $H$ and the last is inversely proportional to $H$. Therefore, the uneven settlement does not monotonously change with height $H$ of the existing embankment like the maximum settlement, but there is an extreme point. Figure 11 shows the corresponding foundation settlement curve when $h=6 \mathrm{~m}$, and $h$ is $1 \mathrm{~m}, 3.5 \mathrm{~m}$, and $6 \mathrm{~m}$, respectively. As can be seen from Figure 11 , when $H=3.5 \mathrm{~m}$, the maximum uneven settlement, that is, the slope of foundation settlement curve is the largest. So far, we can get a comprehensive conclusion about the uneven settlement: the greater the height of the new subgrade, the greater the uneven settlement; and corresponding to a certain height of new subgrade, when the height of the existing foundation is about half of the height of the new foundation $(e \approx 0.5)$, the nonuniform settlement is the largest.

\section{Utilization of Existing Embankments}

In the utilization scheme of existing embankments, the key is the determination of dynamic compaction parameters. Single rammed energy and tamping times are the most important dynamic parameters. Single rammed energy should be determined according to the effective depth of consolidation, tamping times, and the mechanical properties of soil. However, the dynamic compaction of embankment is different from the foundation, the foundation is semiinfinite, and the embankment has a certain size; its corresponding dynamic compaction times not only depend on penetration but also depend on the height of the embankment. From the previous analysis, in order to determine the effective depth of consolidation, we must know the tamping times; in order to determine the tamping times, we must know the effective depth of consolidation. It can be seen that these two problems are coupled together, which brings difficulties to the study of this problem. 


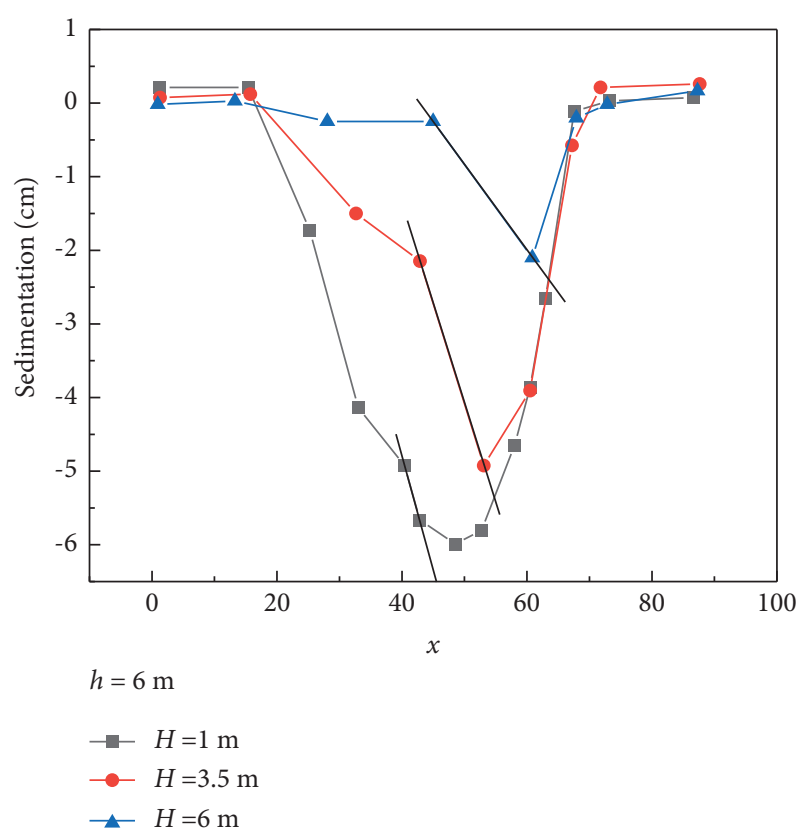

FIgURE 11: The foundation settlement curve corresponding to different existing embankment height.

Previous research and practice on the foundation have shown that the influence of tamping times on the depth of influence is not obvious but increases as the times of tamping and influence depth in the soil had been compacted, when the compaction degree to meet the design requirements becomes the effective reinforcement depth influence depth at this time. In this way, we can ignore the impact of tamping times on the effective depth of consolidation, so that the determination of single rammed energy and the standard of tamping stop are studied separately.

\subsection{Determination of Single Rammed Energy}

5.1.1. Influence Factors. Using Menard formula, the effective reinforcement depth, namely, the embankment height $h$, can be expressed as follows:

$$
h=\alpha \sqrt{\mathrm{MH}},
$$

where $\alpha$ is the coefficient $\left([\mathrm{L}]^{1 / 2}[\mathrm{M}]^{-1 / 2}\right), \mathrm{MH}$ is single rammed energy, $M$ is rammer mass, and $H$ is rammer lifting height.

According to equation (9), single rammed energy can be obtained as follows:

$$
\mathrm{MH}=\left(\frac{h}{\alpha}\right)^{2}
$$

The mechanical parameters of soil are determined by the difference of in situ physical parameters (such as water content and compactness). Fundamentally, $\alpha$ is only related to the mechanical parameters of soil. After many years of operation of existing embankments, the compaction degree of the embankment will change greatly. Assuming that the mechanical parameters of the existing embankment change linearly from top to bottom and assuming that the damping parameters of the soil remain unchanged, the main factors affecting $\alpha$ are as follows:

$$
\alpha=f\left(E_{0}, c_{0}, \varphi_{0}, \rho_{0}, \delta_{E}, \delta_{c}, \delta_{\varphi}, \delta_{\rho}, D\right),
$$

where $E_{0}, c_{0}, \varphi_{0}$, and $\rho_{0}$ are deformation of the embankment surface modulus, cohesive force, internal friction angle, and the wet density, respectively.

Dimensionless equation (11) is obtained:

$$
\alpha \sqrt{\rho_{0}} D=g\left(\frac{c_{0}}{E_{0}}, \frac{\delta_{E} D}{E_{0}}, \frac{\delta_{c} D}{c_{0}}, \frac{\delta_{\varphi} D}{\varphi_{0}}, \frac{\delta_{\rho} D}{\rho_{0}}\right),
$$

where physical meanings of $\delta_{E} D / E_{0}, \delta_{c} D / c_{0}, \delta_{\varphi} D / \varphi_{0}, \delta_{\rho} D / \rho_{0}$ are the ratio of the variation of deformation modulus of soil at $D$ depth, cohesion, internal friction angle, and wet density to each quantity at the surface of embankment, respectively.

5.1.2. Determination of $\alpha$. By means of numerical method, the dynamic response of soil under the action of rammer is analyzed, and the relation between $\alpha$ and each quantity is obtained. Because the times of tamping have little influence on the influence depth, only one dynamic tamping is used in the calculation.

5.1.3. Determination of Reinforcement Depth. In order to obtain the relationship between $\alpha$ and each quantity, the value of $\alpha$ needs to be calculated according to equation (10) from single rammed energy and effective reinforcement depth. The effective reinforcement depth needs to be determined according to the compaction degree, but the compaction degree index cannot be directly obtained by numerical analysis. Therefore, before establishing the relationship between $\alpha$ and various mechanical parameters of soil, the influence depth in numerical calculation should be determined first. After dynamic compaction, embankment soil compaction degree increased; soil body produces a lot of residual strain at the same time; in the case of ignoring soil side, the value of the compaction degree and vertical monotonous geometry corresponding relation in the residual strain is increased. Residual strain can be obtained by numerical calculation. Therefore, the average vertical residual strain in a specific depth range will be used to define the influence depth. The following will take Weiru Road as an example to demonstrate the solution process of the critical mean vertical residual strain.

The actual dynamic tamping condition of Weiru Road was simulated. The single tamping energy was 100 ton $\bullet m$, and the diameter of the tamper hammer $D$ was $2.32 \mathrm{~m}$. The soil parameters were derived from the original mechanical parameters of the existing embankment shown in Table 1. In Table $1, E, c, \varphi$, and $\rho$ are linearly fitted along the depth, so as to get $E_{0}=10.2 \mathrm{MPa}, c_{0}=4.05 \mathrm{kPa},=35.6^{\circ}, \rho_{0}=1.97 \mathrm{~kg} / \mathrm{m}^{3}$, $\delta_{E}=2.42 \mathrm{MPa} / \mathrm{m}, \quad \delta_{c}=2.09 \mathrm{kPa} / \mathrm{m}, \quad \delta_{\varphi}=1.97^{\circ} / \mathrm{m}$, and $\delta_{\rho}=0.04 \mathrm{~kg} / \mathrm{m}^{4}$.

To tamper with dynamic simulation for the process of interaction of embankment, a 10-ton rammer falls from a height of 10 meters, and the $3 \mathrm{D}$ geometric model built is 
shown in Figure 12. Mohr-Coulomb elastoplastic model is adopted in the embankment soil constitutive model. The parameters of $E, c, \varphi$, and $\rho$ show linear variation with depth. The damping of soil adopts local damping, and the damping coefficient is 0.1. In order to simulate infinite soil mass, no reflection boundary is set on all surfaces except the upper surface of embankment.

Figures 13 and 14 show the radial velocity and radial displacement of surface particles, respectively. It can be seen that the radial velocity and displacement increase gradually from inside to outside, reaching the maximum value at about $1.15 \mathrm{~m}$ and then decrease gradually due to the geometric and physical attenuation of surface wave.

Figures 15 and 16 show the vertical velocity and displacement curves of soil particles directly below the rammer. It can be seen that the soil vibration decreases gradually from the top to the bottom, especially in the near surface attenuation; particle vibration velocity $2 \mathrm{~m}$ deep from the surface attenuation is half of the surface velocity. The absolute value of displacement at each point firstly increases from fast to slow with time, and, after reaching the peak value, some rebound occurs due to unloading, and it finally gradually tends to be stable.

The shapes of ramming pits at different times can also be obtained by calculation, as shown in Figure 17. It can be seen that the depth of ramming pit increases at first and then decreases gradually with the surface rebound, and it finally tends to be stable. However, the soil on both sides of the ramming pit has been rising before it is stabilized. The final depth of the ramming pit is about $14 \mathrm{~cm}$ from the surface, which is consistent with the measured data after the field strike.

Figures 18 and 19 show the motion track of a particle at a distance of $1.94 \mathrm{~m}$ and $2.82 \mathrm{~m}$ from the canter of the ram. It can be seen that the particles around the ramming pit move along two tracks successively. According to the time corresponding to the intersection of the two tracks, the particle moves along the second track after the stress wave and the first track before it.

As shown in Figure 20, the average vertical residual strain from the surface to any depth range can be obtained from the calculation results. It can be seen that the average vertical residual strain increases from small to large and then decreases with depth. As the tensile strength of the soil is far less than the compressive strength, the dynamic contact stress at the bottom of the hammer eventually decreases to static pressure, and the unloading wave caused by this easily causes the surface soil to be pulled bad, accompanied by the occurrence of body swelling, so the tensile strain appears near the surface. This is the phenomenon where the surface of embankment becomes loose after dynamic compaction, which further confirms the reason why the soil $30 \mathrm{~cm}$ below the working face is removed during trial compaction. When the average vertical residual strain is calculated from $30 \mathrm{~cm}$ below the surface, it can be found that there is no tensile strain area near the surface, as shown in Figure 20. It is known that the influence depth of embankment corresponding to the above calculation conditions is $4 \mathrm{~m}$, and the critical average vertical residual strain can be obtained from Figure 20 as 0.0382 .

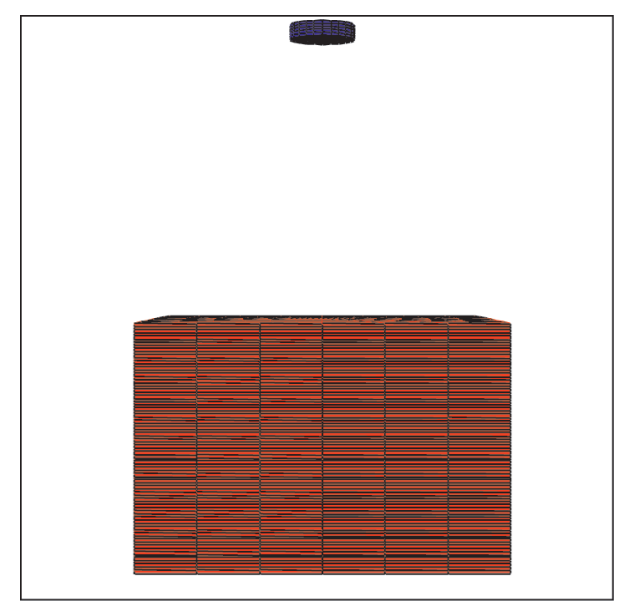

FIGURE 12: Geometrical model.

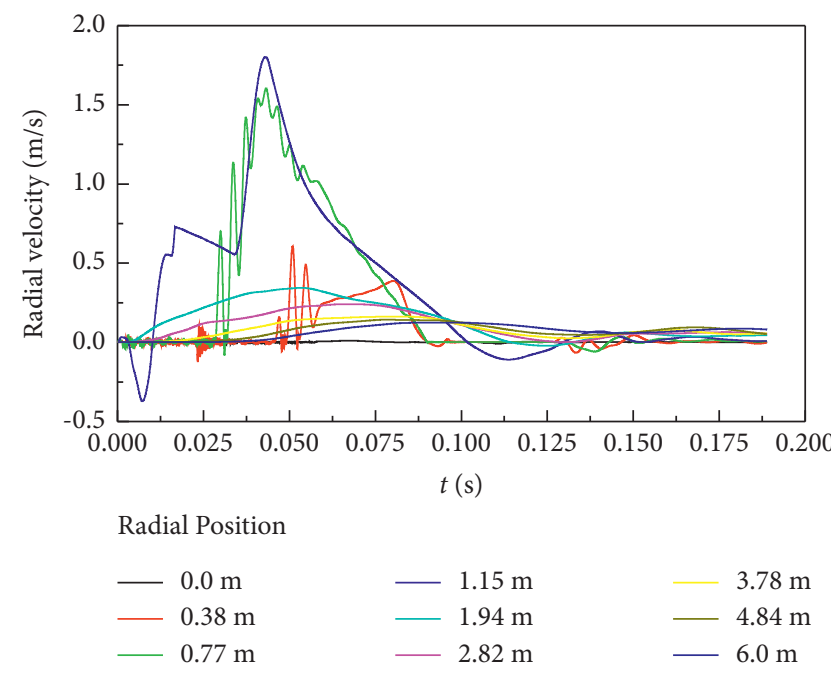

FIGURE 13: Radial velocity of surface particles.

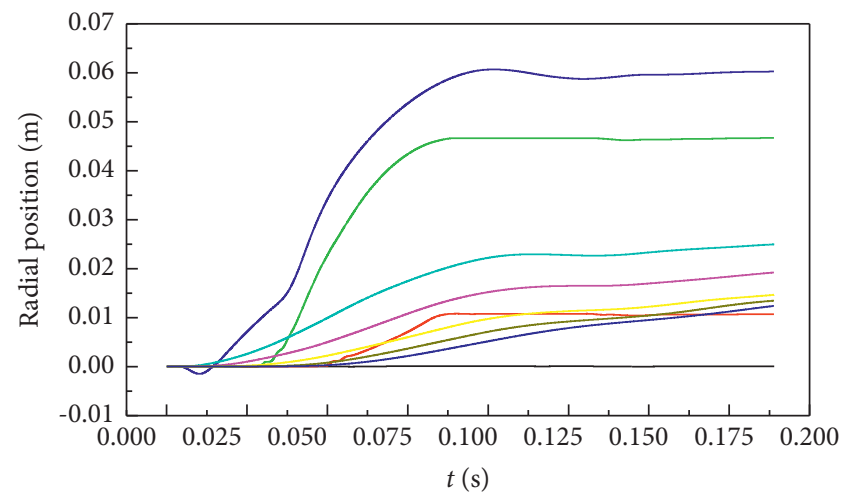

Radial Position

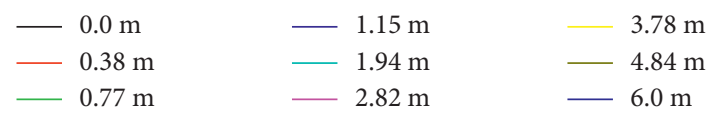

FIgURE 14: Radial displacement of surface particles. 


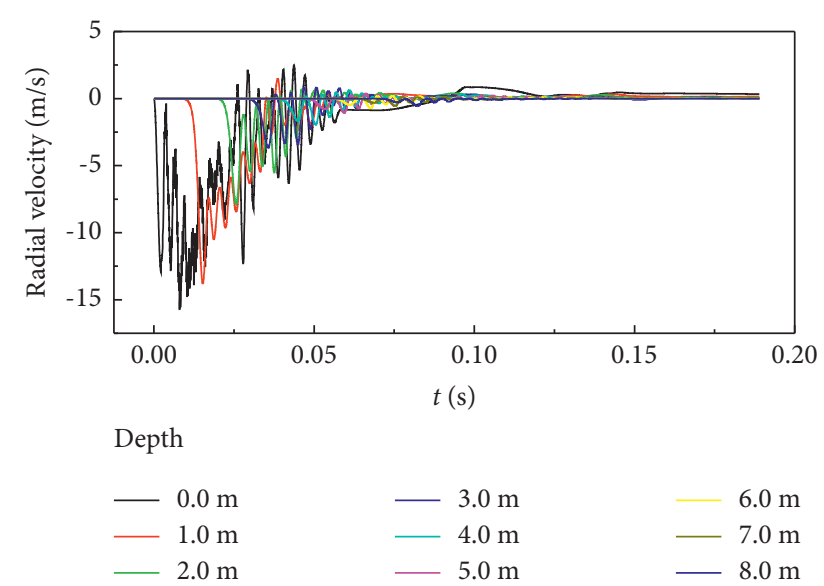

Figure 15: Vertical velocity of soil particles directly below the rammer.

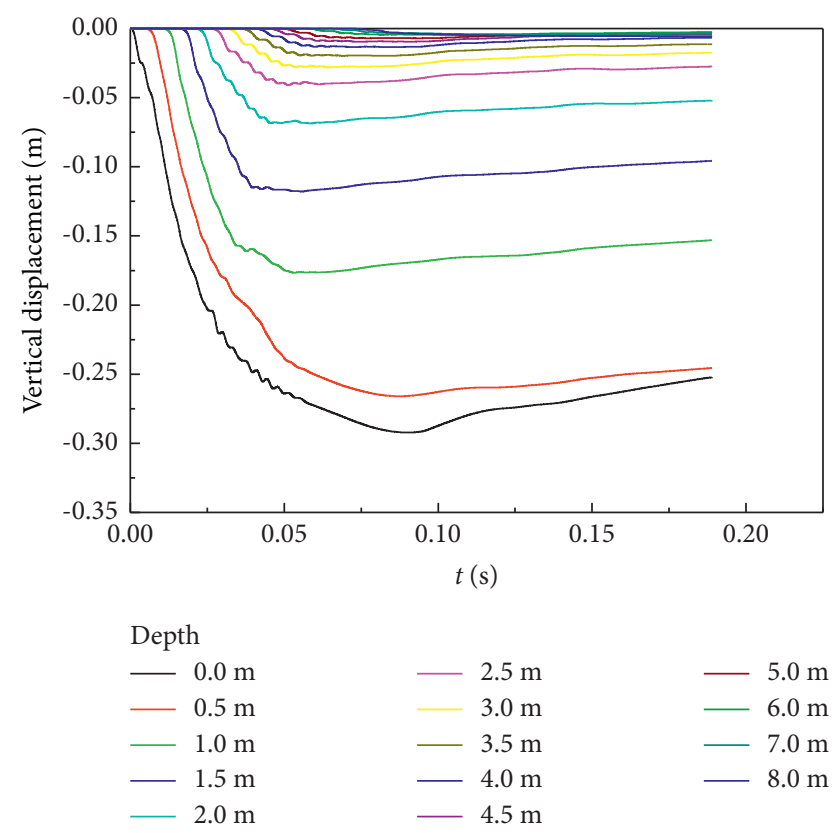

FIGURE 16: Vertical displacement of soil particles directly below the rammer.

In order to fit equation (11), the dynamic calculation as described above is carried out for several working conditions as shown in Table 2. In these working conditions, it is assumed that the initial compaction degree of embankment is not much different from the existing embankment of Weiru Road.

The effective reinforcement depth corresponding to each working condition is obtained from the critical plastic strain 0.0382 , as shown in Table 2. Then $\alpha$ is determined according to the following formula:

$$
\alpha=\frac{h}{\sqrt{\mathrm{MH}}} .
$$

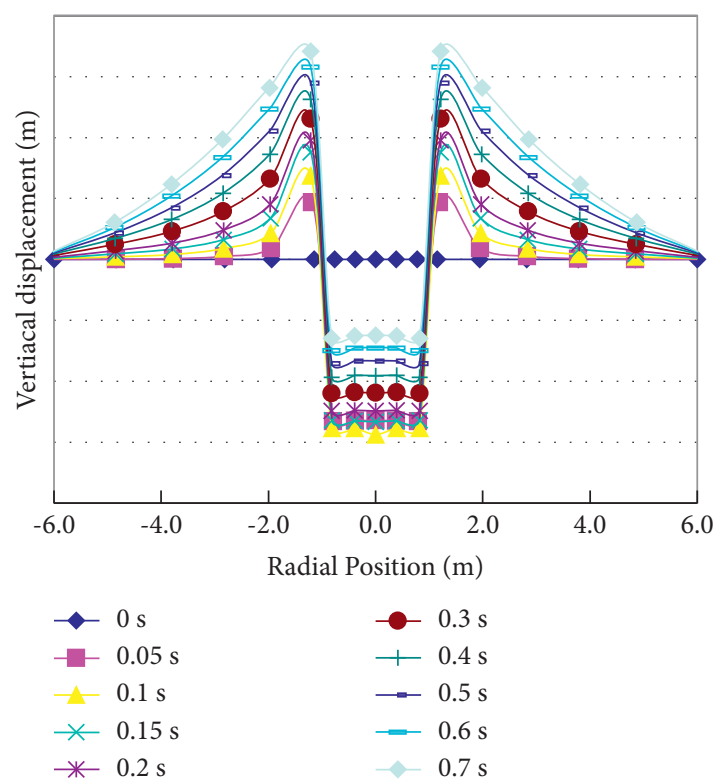

FIgURE 17: Shapes of ramming pits at different times.

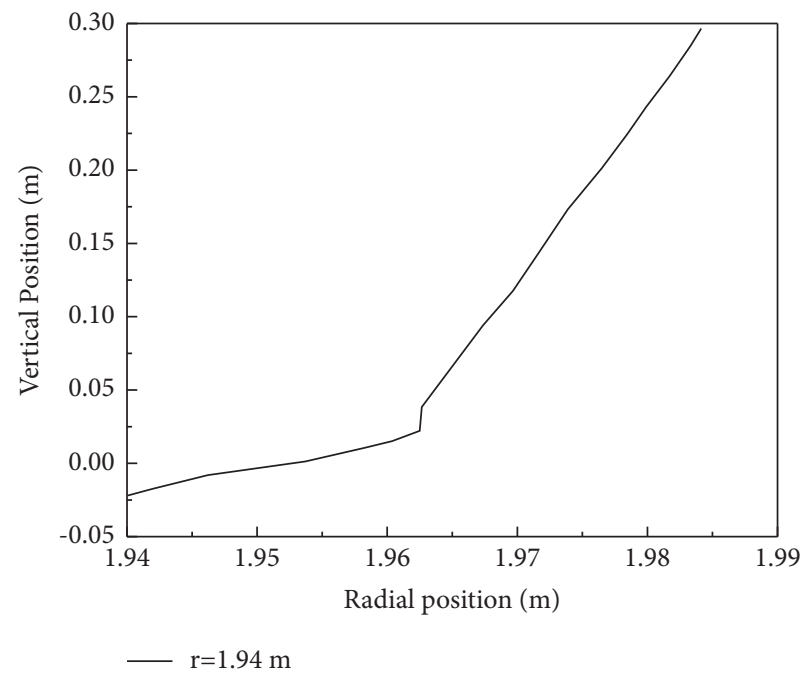

Figure 18: The trajectory of particle at $1.94 \mathrm{~m}$ from the centre of rammer.

Corresponding $\alpha$ of various working conditions is shown in Table 2.

When other parameters remain unchanged, the relationship between dimensionless parameters $c_{0} / E_{0}$ and $\delta_{E} D / E_{0}, \delta_{c} D / c_{0}, \delta_{\varphi} D / \varphi_{0}, \delta_{\rho} D / \rho_{0}$ and dimensionless quantity $\alpha \sqrt{\rho_{0}} D$ is shown in Figures 21-25. It can be seen that only $c_{0} / E_{0}$ and $\alpha \sqrt{\rho_{0}} D$ are in line with the hyperbolic relationship as shown in Figure 21, and the other quantities are linear with $\alpha \sqrt{\rho_{0}} D$. Therefore, the following equation can be used to fit equation (8): 


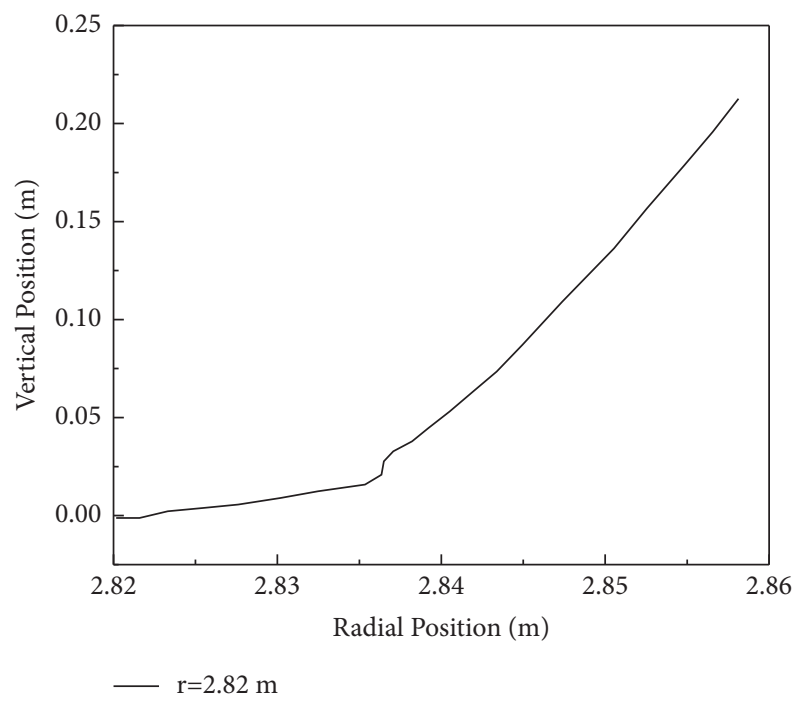

Figure 19: The trajectory of particle at $2.82 \mathrm{~m}$ from the centre of rammer.

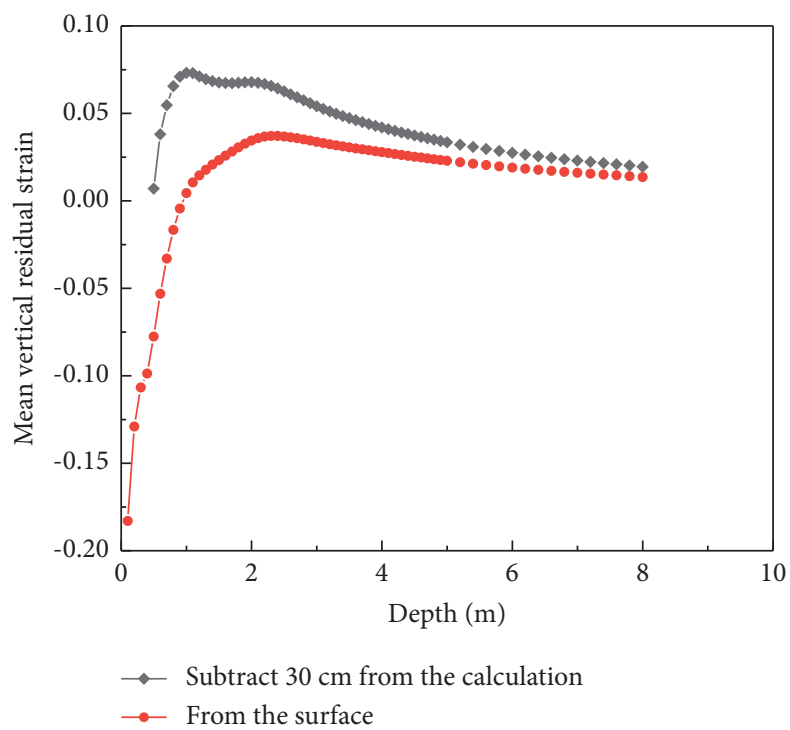

FIgURE 20: Curve of average vertical residual strain depth.

$$
\alpha \sqrt{\rho_{0}} D=P_{0}+P_{1} \frac{E_{0}}{c_{0}}+P_{2} \frac{\delta_{E} D}{E_{0}}+P_{3} \frac{\delta_{c} D}{c_{0}}+P_{4} \frac{\delta_{\varphi} D}{\varphi_{0}}+P_{5} \frac{\delta_{\rho} D}{\rho_{0}}
$$

where $P_{i}$ represents fitting parameters.

The fitting parameters are shown in Table 3. To investigate the fitting effect, the calculated value of $\alpha \sqrt{\rho_{0}} D$ and fitting value of equation (10) are drawn in the same diagram, as shown in Figure 26. It can be seen that, except for a few points, most of the data points fall near the line $y=x$, indicating that the fitting effect of equation (10) meets the engineering requirements.

5.2. Compaction Stopping Standard. From the macroscopic aspect, the compaction degree of embankment is mainly related to the effective reinforcement depth (i.e., embankment height) and penetration degree, namely,

$$
d_{c}=f(h, s)
$$

Make the above formula dimensionless and get

$$
d_{c}=F\left(\frac{s}{h}\right)
$$

The physical significance of $s / h$ is the average residual plastic strain of the embankment in the last two tampings.

Assume that the first layer $i$ compaction $d_{c i}$ and $s / h$ conforms to the linear relationship as follows:

$$
d_{c i}=a_{i} \frac{s}{h}
$$

where $a_{i}$ is corresponding to the first $i$ layer coefficient.

Under any condition, degree of compaction should meet the specification requirements; namely, $d_{c i}$ remains 
TABLE 2: Summary of calculation conditions.

\begin{tabular}{|c|c|c|c|c|c|c|c|c|c|c|c|c|}
\hline $\begin{array}{l}\text { Working } \\
\text { condition }\end{array}$ & & $\delta \rho / \rho_{0}$ & & $\delta_{\mathrm{c}} / c_{0}$ & & $\delta_{\mathrm{E}} / E_{0}$ & & $\delta_{\phi} / \phi_{0}$ & & $c_{0} / E_{0}$ & $h$ & $\alpha$ \\
\hline 1 & & 0.020 & & -0.516 & & -0.237 & & 0.055 & & 0.397 & 4 & 0.01265 \\
\hline 2 & 0.010 & $\begin{array}{c}\delta \rho=0.02 \\
\rho_{0}=2.0\end{array}$ & & -0.516 & & -0.237 & & 0.055 & & 0.397 & 3.8156 & 0.01207 \\
\hline 3 & 0.030 & $\begin{array}{c}\delta \rho=0.06 \rho \\
0=2.0\end{array}$ & & -0.516 & & -0.237 & & 0.055 & & 0.397 & 3.659 & 0.01157 \\
\hline 4 & 0.015 & $\begin{array}{c}\delta \rho=0.03 \rho \\
0=2.0\end{array}$ & & -0.516 & & -0.237 & & 0.055 & & 0.397 & 3.836 & 0.01213 \\
\hline 5 & 0.025 & $\begin{array}{c}\delta \rho=0.05 \\
\rho_{0}=2.0\end{array}$ & & -0.516 & & -0.237 & & 0.055 & & 0.397 & 3.906 & 0.01235 \\
\hline 6 & & 0.020 & -0.260 & $\begin{array}{c}\delta_{\mathrm{c}}=-1.05 \\
c_{0}=4.05\end{array}$ & & -0.237 & & 0.055 & & 0.397 & 4.352 & 0.01376 \\
\hline 7 & & 0.020 & -0.130 & $\begin{array}{c}\delta_{\mathrm{c}}=-0.53 \\
c_{0}=4.05\end{array}$ & & -0.237 & & 0.055 & & 0.397 & 4.546 & 0.01438 \\
\hline 8 & & 0.020 & 0.260 & $\begin{array}{l}\delta_{\mathrm{c}}=1.05 \\
c_{0}=4.05\end{array}$ & & -0.237 & & 0.055 & & 0.397 & 5.292 & 0.01673 \\
\hline 9 & & 0.020 & 0.120 & $\begin{array}{c}\delta_{\mathrm{c}}=0.5 \\
c_{0}=4.05\end{array}$ & & -0.237 & & 0.055 & & 0.397 & 5.286 & 0.01672 \\
\hline 10 & & 0.020 & & -0.516 & -0.120 & $\begin{array}{c}\delta_{\mathrm{E}}=-1.21 \\
E_{0}=10.2\end{array}$ & & 0.055 & & 0.397 & 4.178 & 0.01321 \\
\hline 11 & & 0.020 & & -0.516 & -0.360 & $\begin{array}{c}\delta_{\mathrm{E}}=-3.63 \\
E_{0}=10.2\end{array}$ & & 0.055 & & 0.397 & 3.818 & 0.01207 \\
\hline 12 & & 0.020 & & -0.516 & 0.060 & $\begin{array}{l}\delta_{\mathrm{E}}=0.61 \\
E_{0}=10.2\end{array}$ & & 0.055 & & 0.397 & 4.376 & 0.01384 \\
\hline 13 & & 0.020 & & -0.516 & 0.100 & $\begin{array}{l}\delta_{\mathrm{E}}=1.02 \\
E_{0}=10.2\end{array}$ & & 0.055 & & 0.397 & 4.466 & 0.01412 \\
\hline 14 & & 0.020 & & -0.516 & & -0.237 & 0.028 & $\begin{array}{c}\delta_{\phi}=0.985 \\
\phi_{0}=35.6\end{array}$ & & 0.397 & 1.14 & 0.0036 \\
\hline 15 & & 0.020 & & -0.516 & & -0.237 & 0.042 & $\begin{aligned} \delta_{\phi} & =1.5 \phi \\
0 & =35.6\end{aligned}$ & & 0.397 & 2.56 & 0.0081 \\
\hline 16 & & 0.020 & & -0.516 & & -0.237 & 0.049 & $\begin{array}{c}\delta_{\phi}=1.75 \phi \\
0=35.6\end{array}$ & & 0.397 & 1.4 & 0.00443 \\
\hline 17 & & 0.020 & & -0.516 & & -0.237 & 0.070 & $\begin{aligned} \delta_{\phi} & =2.5 \phi \\
0 & =35.6\end{aligned}$ & & 0.397 & 3.524 & 0.01114 \\
\hline 18 & & 0.020 & & -0.516 & & -0.237 & & 0.055 & 0.1 & $\begin{aligned} c_{0} & =1.02 \delta \\
c & =-0.53\end{aligned}$ & 7.989 & 0.02526 \\
\hline 19 & & 0.020 & & -0.516 & & -0.237 & & 0.055 & 0.2 & $\begin{array}{c}c_{0}=2.025 \delta \\
c=-1.05\end{array}$ & 4.866 & 0.01539 \\
\hline 20 & & 0.020 & & -0.516 & & -0.237 & & 0.055 & 0.8 & $\begin{array}{c}E_{0}=5.1 \\
\delta_{\mathrm{E}}=-1.21\end{array}$ & 3.044 & 0.00963 \\
\hline 21 & & 0.020 & & -0.516 & & -0.237 & & 0.055 & 0.6 & $\begin{array}{c}E_{0}=6.8 \\
\delta_{\mathrm{E}}=-1.61\end{array}$ & 2.676 & 0.00846 \\
\hline
\end{tabular}

Note. $\delta \rho\left(\mathrm{g} / \mathrm{cm}^{3} / \mathrm{m}\right), \rho_{0}\left(\mathrm{~g} / \mathrm{cm}^{3}\right), \delta_{\mathrm{c}}(\mathrm{kPa} / \mathrm{m}), c_{0}(\mathrm{kPa}), \delta_{E}(\mathrm{MPa} / \mathrm{m}), E_{0}(\mathrm{MPa}), \delta_{\phi}\left({ }^{\circ} / \mathrm{m}\right), \phi_{0}\left({ }^{\circ}\right), h(\mathrm{~m})$, and $\alpha\left(\mathrm{m}^{0.5} / \mathrm{kg}^{0.5}\right)$.

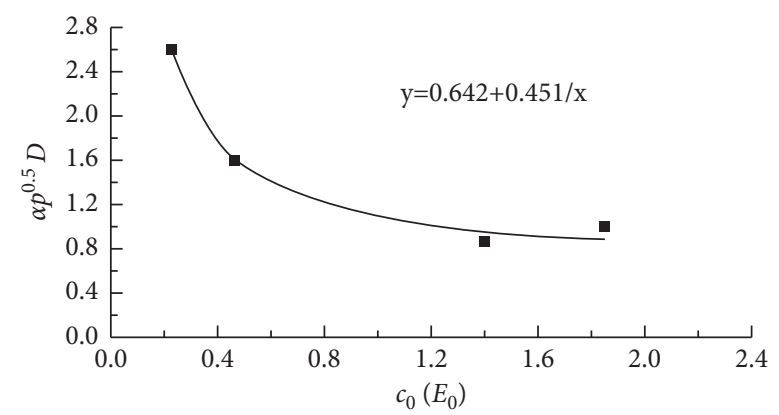

Figure 21: Curve of $\sqrt{\rho_{0}} D \sim c_{0} / E_{0}$. 


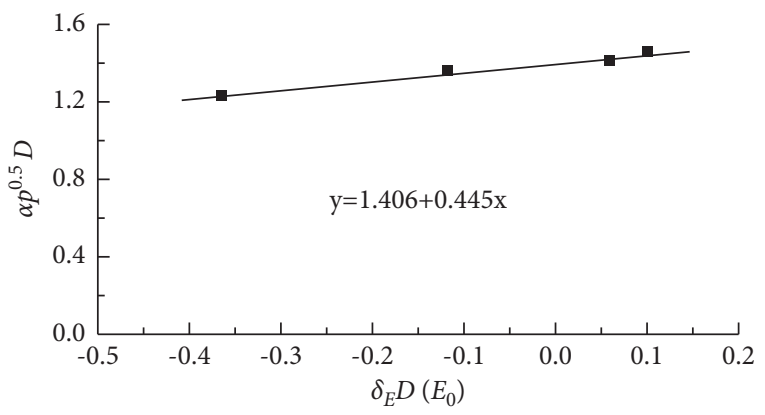

Figure 22: Curve of $\alpha \sqrt{\rho_{0}} D \sim \delta_{E} D / E_{0}$.

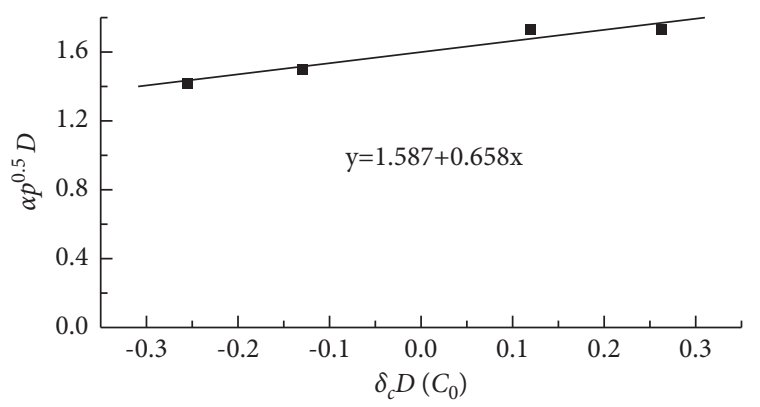

Figure 23: Curve of $\sqrt{\rho_{0}} D \sim \delta_{c} D / c_{0} \sim \delta_{c} D / c_{0}$.

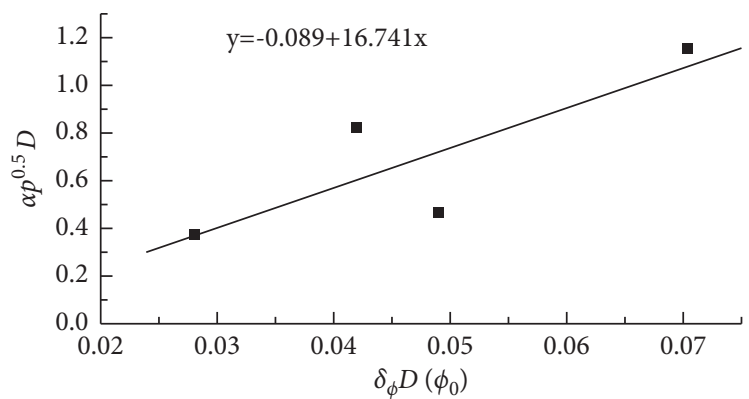

Figure 24: Curve of $\alpha \sqrt{\rho_{0}} D \sim \delta_{\varphi} D / \varphi_{0}$.

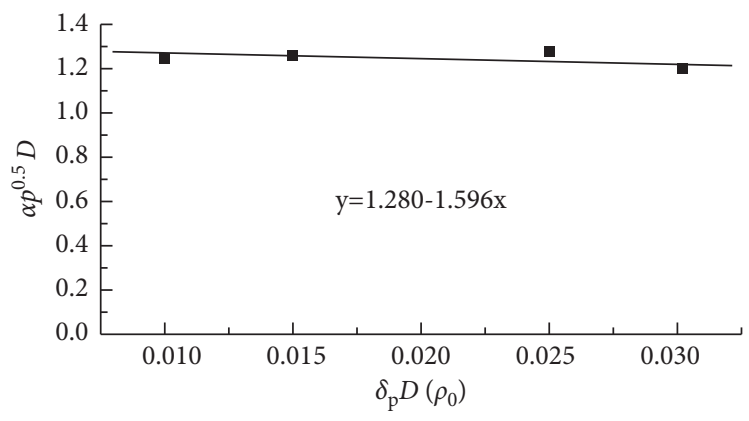

Figure 25: Curve of $\alpha \sqrt{\rho_{0}} D \sim \delta_{\rho} D / \rho_{0}$. 
TABLE 3: Fitting parameters.

\begin{tabular}{lcccccc}
\hline$P_{i}$ & $P_{0}$ & $P_{1}$ & $P_{2}$ & $P_{3}$ & $P_{4}$ & $P_{5}$ \\
\hline Parameter & -0.04892 & 0.44786 & 0.37909 & 0.36068 & 10.78445 & -0.68787 \\
\hline
\end{tabular}

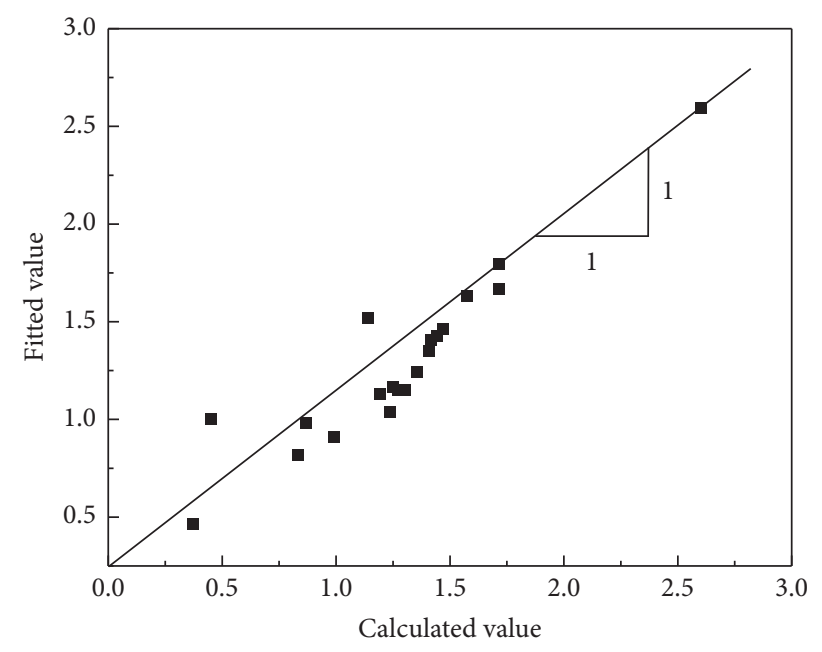

FIGURE 26: Fitting effect drawing.

unchanged, while $a_{i}$ is constant, so, under any condition, $s / h$ is also the same. In the trial ramming of Weiru Road, $h=4 \mathrm{~m}$ and $s=0.05 \mathrm{~m}$, so

$$
s=0.0125 h
$$

In dynamic compaction of embankment, penetration as shown in equation (18) is used as the control standard. Equation (18) shows that penetration is related to embankment height, and it is incorrect to use fixed penetration as the standard for compaction.

\section{Conclusions}

Using the finite difference method, the numerical analysis results of the consolidation and settlement law of the ground caused by the transformation of grade road into expressway show the following:

(1) In the process of road reconstruction, the size of foundation consolidation settlement is related to the widening mode and the height of the existing and new embankment. If the foundation and subgrade are not treated, the settlement of consolidation exceeds the requirements of the code, and the difference of settlement is more obvious at the junction of existing and new embankment. The settlement difference at the junction of existing and new embankment causes tensile damage on the surface of roadbed, which leads to longitudinal cracks and other diseases. The existence of the existing embankment makes the soil particles of the embankment move to the new foundation. The settlement difference of embankment after construction exceeds the bearing capacity of pavement structure, so engineering measures should be taken to reinforce the existing embankment and the new natural foundation and focus on the joint of the existing and new embankment.

(2) The settlement curve of foundation shows obvious asymmetry, which forms a great deal of uneven settlement at the junction of new and existing subgrade. With the increase of load eccentricity, the position of the maximum settlement point of foundation gradually moves to the new foundation, so the treatment of the new foundation is an important measure to reduce the settlement of foundation. The greater the height of the new subgrade is, the greater the uneven settlement is. For a certain height of new subgrade, when the height of existing subgrade is about half, the uneven settlement is the largest. In order to reduce the uneven settlement of foundation, it is suggested to strengthen the new foundation.

(3) In order to put forward the dynamic compaction parameters suitable for general embankment, the dynamic compaction results of existing embankment are extended. Single rammed energy of general embankment can be shown in the following equation:

$$
\mathrm{MH}=\left(\frac{h}{\alpha}\right)^{2}
$$

where $\quad \alpha=g\left(c_{0} / E_{0}, \delta_{E} D / E_{0}, \delta_{c} D / c_{0}, \delta_{\varphi} D / \varphi_{0}, \delta_{\rho} D /\right.$ $\left.\rho_{0}\right) /\left(\sqrt{\rho_{0}} D\right)$; if the soil is constant, $\alpha$ has a constant value, and $h$ is the height of the embankment. 
(4) Taking practical engineering as an example, the general compaction stopping standard expressed by penetration $s$ degree is presented by numerical method, namely, $s=0.0125 \mathrm{~h}$.

\section{Data Availability}

No data were used in this study.

\section{Conflicts of Interest}

The authors declare that they have no conflicts of interest.

\section{References}

[1] The Highways Agency, The Scottish Office Development Department, the Welsh Office, the Department of the Environment for Northern Ireland, Geotechnical Considerations and Techniques for Widening Highway Earthworks, 1991.

[2] Y. L. Chen, Y. Lu, Z. N. Zhang et al., "The cause of longitudinal pavement fissures and preventions on highway road-widening engineering," East China Highway, vol. 40, no. 1, pp. 38-41, 2003.

[3] Y. S. Chen and Z. Y. Liu, "Consideration on highway reconstruction and expansion plan," Highway, vol. 7, no. 7, 2001.

[4] J. L. Yu, X. Q. Chen, F. Li et al., "Study on settlement characteristics and influencing factors of longitudinal heightening and widening roadbed of reconstructed and expanded roads," Subgrade Engineering, vol. 40, no. 9, pp. 35-39, 2019.

[5] N. Jia, R. P. Chen, Y. M. Chen, and L. X. Xu, "Theoretical analysis and measurement for widening project of hang-yong expressway," Chinese Journal of Geotechnical Engineering, vol. 25, no. 6, pp. 755-760, 2004.

[6] S. Li, D. L. Zhang, Q. Fang et al., "Research on differential settlement control standard for road bilateral widening project," Journal of Highway and Transportation Research and Development, vol. 31, no. 5, pp. 16-20, 2014.

[7] H. G. B. Allersma, L. Ravenswawy, and E. Vos, "Investigation of road widening on so $\mathrm{ft}$ soil using a small centrifuge," Transportation Research Record, 1994.

[8] E. Vos, J. F. Couvreur, and M. Vermaut, "Comparison of numerical analysis with field data of a road widening project on peaty soil," in Proceedings of the International Workshop: Advance in understanding and modelling the mechanical behavior of peat, pp. 267-274, Balkema, Rotterdam, 1994.

[9] Z. L. Zhou and J. L. Zheng, "Finite element analysis in the design of old road widening," Mechanics in Engineering, vol. 17 , no. 5, pp. 18-20, 1995.

[10] J. S. Qian and J. M. Ling, "Analysis of mechanical responses for highway-widening engineering on soft soil foundation," Chinese Journal of Underground Space and Engineering, vol. 6, no. 3, pp. 481-486, 2010.

[11] Y. S. Li, J. G. Zhang, J. L. Zhao et al., "Study on differential settlement of subgrade widening based on FlAC3D," Quality Measurement and Control, vol. 143, no. 6, pp. 143-148, 2020.

[12] Q. L. Huang, J. M. Ling, B. M. Tang et al., "Experimental research on incoordinate deformation between existing subgrade and the widening one," Journal of Highway and Transportation Research and Development, vol. 21, no. 12, pp. 18-21, 2004.

[13] X. Weng and W. Wang, "Influence of differential settlement on pavement structure of widened roads based on large-scale model test," Journal of Rock Mechanics and Geotechnical Engineering, vol. 3, no. 1, pp. 90-96, 2011.

[14] L. Chen, H. L. Liu, and Y. H. Chen, "Numerical analysis of foundation improvement effect for expressway widening engineering," Rock and Soil Mechanics, vol. 27, no. 11, pp. 2066-2070, 2006.

[15] J. H. Zhang, A. S. Zhang, C. Huang, and H. Yu, "Characterising the resilient behaviour of pavement subgrade with construction and demolition waste under Freeze-Thaw cycles," Journal of Cleaner Production, vol. 300, Article ID 126702, 2021.

[16] J. Zhang and F. Li, "Numerical simulation of the moisture migration of unsaturated clay embankments in southern China considering stress state," Bulletin of Engineering $\mathrm{Ge}$ ology and the Environment, vol. 80, no. 1, pp. 11-24, 2021.

[17] J. Zhang, J. Peng, J. Li, and A. Zhang, "Prediction of permanent deformation for subgrade soils under traffic loading in Southern China," International Journal of Pavement Engineering, pp. 1-10, 2020.

[18] H. Yang, "Design thinking and construction control of soft soil foundation in reconstruction and extension construction of the shen-da expressway," Northeastern Highway, vol. 25, no. 2, pp. 11-14, 2002.

[19] P. Nie, X. J. Qu, F. Q. Liu et al., “Allowable differential settlement for widened roadbed in shen-da expressway reconstruction," Journal of Highway and Transportation Research and Development, vol. 22, no. 11, pp. 18-20, 2005.

[20] X. X. Cheng, X. C. Wang, and Z. W. Gao, "Control criterion of subgrade differential settlement based on pavement damage response," Journal of Chang'an University (Natural Science Edition): Natural Science Edition, vol. 30, no. 5, pp. 31-34, 2010.

[21] Q. S. Zhao, G. S. Zhao, C. H. Zhang et al., "Threshold of settlement control for widened jing-shi embankments," Journal of Liaoning Technical University, vol. 31, no. 1, pp. 69-72, 2012. 